\title{
1 Wiring diagram of the oxytocin system in the mouse brain
}

2

3 Seoyoung Son ${ }^{1}$, Steffy B. Manjila ${ }^{1}$, Kyra T. Newmaster ${ }^{1}$, Yuan-ting Wu ${ }^{1}$, Daniel J. Vanselow ${ }^{2}$,

4 Matt Ciarletta $^{1}$, Keith C. Cheng ${ }^{2}$, and Yongsoo Kim ${ }^{1}$

5

61 Department of Neural and Behavioral Sciences, Penn State University, Hershey, PA, USA

72 Department of Pathology, Penn State University, Hershey, PA, USA

8

11 Corresponding author:

12 Yongsoo Kim

13 Email: yuk17@psu.edu

14

15 Keyword: Oxytocin, Oxytocin receptor, Brain mapping, synaptic input, axonal output,

16 anatomical connectivity, serial two-photon tomography, connectome 


\section{Abstract}

In the brain, oxytocin (OT) neurons make direct connections with discreet regions to regulate social behavior and diverse physiological responses. Obtaining an integrated neuroanatomical understanding of pleiotropic OT functions requires comprehensive wiring diagram of OT neurons. Here, we have created a whole-brain map of distribution and anatomical connections of hypothalamic OT neurons, and their relationship with OT receptor (OTR) expression. We used our brain-wide quantitative mapping at cellular resolution combined with a $2 \mathrm{D}$ flatmap to provide an intuitive understanding of the spatial arrangements of OT neurons. Then, we utilized knock-in Ot-Cre mice injected with Cre dependent retrograde monosynaptic rabies viruses and anterograde adeno associated virus to interrogate input-output patterns. We find that brain regions with cognitive functions such as the thalamus are reciprocally connected, while areas associated with physiological functions such as the hindbrain receive unidirectional outputs. Lastly, comparison between OT output and OTR expression showed no significant quantitative correlation, suggesting that OT transmission mostly occurs through indirect pathways. In summary, our OT wiring diagram provides structural and quantitative insights of distinct behavioral functions of OT neurons in the brain.

\section{Significance Statement}

Oxytocin (OT) neurons in the brain play an important role in socio-physiological responses. Impairment of OT signaling has been implicated in many neurodevelopmental disorders. To understand diverse OT functions in the context of discreet neural circuits, it is imperative to understand the anatomical arrangement of OT neurons across the whole brain in significant detail. Here, we have established a comprehensive brain-wide wiring diagram of OT neurons. Our anatomical and connectivity map of OT neurons includes brain-wide cell distribution, synaptic inputs, axonal outputs, and their relationships with the oxytocin receptor expression. This whole brain structural perspective of the OT system provides a foundation for understanding the diversity of neural circuits modulated by OT and will guide future circuitbased OT functional studies. 


\section{Introduction}

52 Oxytocin (OT) is a highly conserved neuropeptide, playing key roles in regulating social

53 behavior and other physiological functions (1-4). Impairment in OT signaling has been heavily implicated in many neurodevelopmental disorders including autism $(5,6)$. Correcting OT signaling is being pursued as a potential therapy to alleviate social behavioral symptoms in many brain disorders (7), but our limited neurobiological understanding of OT signaling in the brain hampers the development of targeted approaches aimed at altering specific OT functions without affecting other biological pathways. A comprehensive anatomical understanding of OT neurons would enable integrated neural circuit specific studies to decipher the neural substrate of distinct

60 OT functions.

61 The majority of OT producing neurons are located in the paraventricular nucleus of the hypothalamus (PVH) and the supraoptic nucleus (SO) while fewer OT neurons reside in the bed nucleus of the stria terminalis (BST), the medial amygdala (MEA), and the accessory nuclei (AC) (8). OT neurons receive input from distinct brain regions [e.g., the thalamus (TH)] to integrate sensory input and internal information to release OT in a context dependent manner in order to modulate specific downstream circuitry $(9,10)$. The actions of OT are mainly mediated by a single subtype of the OT receptor (OTR) expressed in distinct brain regions as well as peripheral tissues (11-13). In addition to the well-known peripheral release of OT as a hormone via the posterior pituitary, OT neurons send direct projections to discreet brain areas that frequently express OTR, thereby modulating circuit specific functions. For example, OT signaling is linked with the medial prefrontal cortex for social cognition $(14,15)$, CA2 of the hippocampus for social memory $(16,17)$, the central amygdala for fear modulations $(18,19)$, the parabrachial nucleus (PB) for fluid intake (20), and the spinal cord for pain perception $(21,22)$. Despite these prior studies, we still lack a quantitative and comprehensive wiring diagram of the OT neurons in a standard 3D reference brain. Moreover, quantitatively characterizing the wiring relationships between OT neurons and OTR expression patterns across the whole brain is made difficult due to the need to simultaneously examine microscopic structures (e.g., cell bodies and their axonal projections) in a macroscopic structure, namely the whole brain.

Here, we use a high-resolution quantitative brain mapping method in combination with cell type specific transgenic mice and viral tools to establish the wiring diagram of OT neurons in the mouse brain. We devised a 2D hypothalamic flatmap to quantify OT distribution, and analyzed synaptic inputs and long-range output patterns of hypothalamic OT neurons. We find reciprocally and unidirectionally connected brain regions with OT neurons, linked with distinct cognitive and physiological functions, respectively. Moreover, systemic comparisons between the OT projectome and OTR expression reveal potential brain regions with direct synaptic or non-synaptic OT signaling. The imaging data are mapped onto a standard reference brain to facilitate cross-examination (23) and freely available via user-friendly web visualizations at 


\section{Results}

\section{Quantitative expression of oxytocin neurons in the mouse brain}

Although the location of OT neurons in the mouse hypothalamus (HY) is relatively wellestablished (8), data showing quantitative brain-wide OT distribution in complex 3D structures remains elusive. To examine the anatomical distribution of OT neurons across the whole brain, we used heterozygote OT knock-in mice with Cre recombinase ( $\mathrm{Ot}$ - $\mathrm{Cre})$ crossed with Ai14 reporter mice (OT:Ai14) (24). We imaged the entire mouse brain at cellular resolution using serial two-photon tomography (STPT) and performed quantitative mapping using previously established computational methods ( 25$)(\mathrm{n}=8$ brains, 3 males, 3 virgin females, and 2 lactating females, Fig. 1A-B, Movie S1). There was no significant difference between male, virgin female, and lactating female (Table S1), which is consistent with an earlier study (18). The majority of OT neurons ( $42 \%, 792$ out of total 1896 cells) were expressed in the PVH regions (PVH, descending division of $\mathrm{PVH}$, anterior, intermediate, and preoptic part of periventricular hypothalamic nucleus, and subparaventricular zone) followed by the SO, the tuberal nucleus (TU), the MEA, and the BST (Table S1). The rest of the OT neurons are expressed in 10 different brain regions (Table S1). The Cre-driven reporter system used in the present study permanently labels all Cre positive cells including developmentally transient expression (11). To distinguish neurons actively expressing OT in adult from developmentally labeled cells, we performed immunohistochemistry using an OT antibody in OT:Ai14 mice. We confirmed that almost all OT immuno positive neurons $(97 \%, 1733$ out of 1790 cells, $n=4)$ were labeled by tdTomato from OT:Ai14 mice (Fig. S1). In contrast, $76 \%$ of tdTomato labeled cells were OT immuno positive ( 1733 out of 2277 cells) in the PVH. Smaller portions of tdTomato cells in the SO ( $44 \%, 654$ out of 1508 cells) and the MEA ( $8 \%, 31$ out of 375 cells) retain active OT expression (Fig. S1). This suggests that OT neurons in different brain regions undergo differential rates of developmental down-regulation in the adult brain (26). To further visualize the spatial expression pattern of OT neurons, we created a 2D hypothalamic flatmap. Evenly spaced bins provide a flattened 2D spatial unit to quantify and to display signals from the $3 \mathrm{D}$ brain (Fig. 1C-D). The 2D hypothalamic flatmap was delineated with Allen Common Coordinate Framework (CCF) and Franklin-Paxinos atlas based anatomical labels $(23,27,28)$ (Fig 1C). The regional boundaries of the two labeling systems generally agreed with each other in the major OT expressing regions (e.g., the $\mathrm{PVH}$ and the $\mathrm{SO}$ ) despite noticeable discrepancies in the ventricle hypothalamic area (e.g., the TU) (Fig 1C-D) (28). The density heatmap on the hypothalamic flatmap provides an intuitive and quantitative display of the regionally heterogeneous distribution of OT neurons (Fig. 1D). We also provide detailed distribution patterns of the OT neurons within the PVH using a 2D PVH flatmap (Fig. S2). Together, these data provide the quantitative expression pattern of OT neurons throughout the whole brain. 

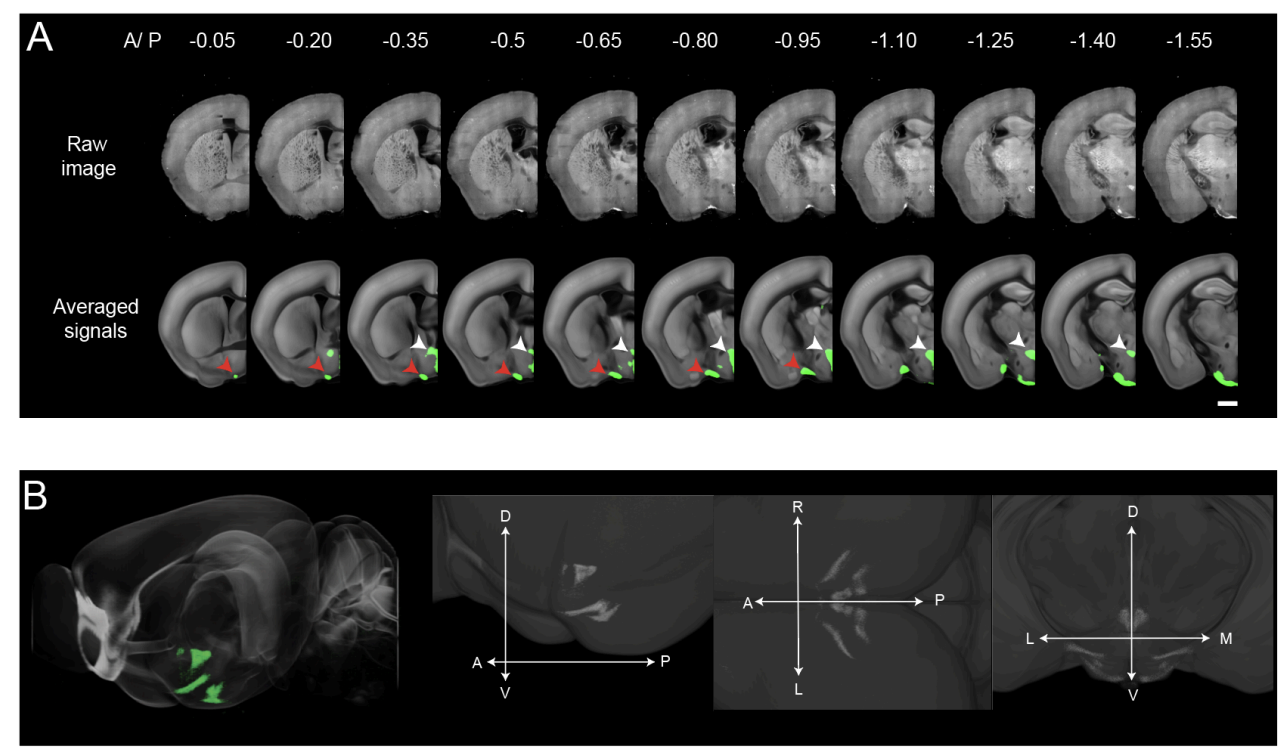

C

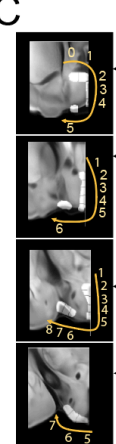

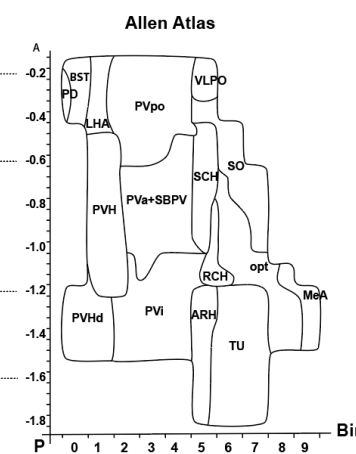

Franklin-Paxinos Atlas

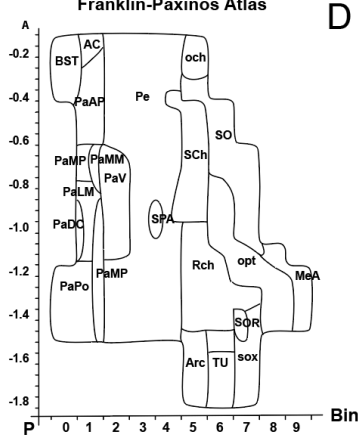

D

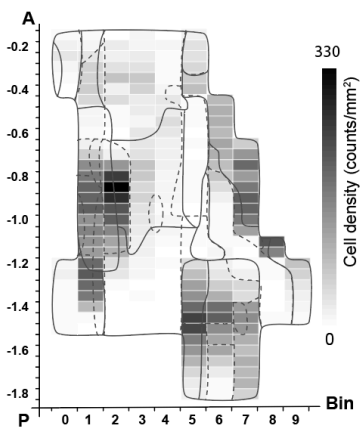

Figure 1. Distribution of oxytocin neurons.

(A) Signals from OT:Ai14 across representative coronal planes of the mouse brain. (top) STPT images registered onto the Allen CCF reference brain, (bottom) averaged signals ( $\mathrm{n}=8$, green dots) overlaid on the reference brain. White and red arrowheads indicate the PVH and SO, the separately. Scale bar $=1 \mathrm{~mm}$. See also Movie S1, Table S1. (B) 3D distribution of oxytocin neurons. A/P: anterior/posterior, L/M:

Lateral/medial, D/V: dorsal/ventral. (C) 2D hypothalamic flatmaps. Small inserts with coronal sections illustrate how bins (while areas with numbers) were generated at different coronal planes. Anatomical labels in the flatmap are delineated based on Allen mouse brain atlas (left) and Franklin-Paxinos atlas (right). The $\mathrm{X}$-axis is for bin numbers and $\mathrm{Y}$-axis is for the bregma $\mathrm{A} / \mathrm{P}$ axis. (D) Heatmap of oxytocin neuronal density in the overlay of Allen and Franklin-Paxinos labels in solid and dotted lines, respectively. The full name of abbreviations can be found in Table S1.

\section{Monosynaptic inputs to oxytocin neurons}

Previous studies suggest that the PVH area receives input from specific brain areas such as the dorsomedial hypothalamus area, the medial preoptic area (MPO), the subparafascicular area, and the posterior intralaminar nuclei to integrate external stimuli and internal state (29-31). To map brain-wide trans-synaptic inputs in a cell type specific manner, we injected monosynaptic retrograde rabies viruses into the $\mathrm{PVH}$ and the SO of the $\mathrm{Ot}$-Cre knock-in mice (32). We used our qBrain mapping method to quantify input neurons throughout the whole brain (25). To capture total input to each anatomical area, we combined input signals from multiple independent injections targeting a specific brain region and overlaid them onto a reference brain 
while displaying input neurons with pseudo-colored green for the $\mathrm{PVH}$ ( $\mathrm{n}=6$ brains) and red for the SO ( $\mathrm{n}=4$ brains) (Fig. 2A, Movie S2). Our analysis reveals that more than 50 different structures provide inputs to OT neurons (Fig. 2B). Both the $\mathrm{PVH}$ and the $\mathrm{SO}$ receive major inputs from other hypothalamic brain regions. Overall, PVH OT neurons take inputs from more regions than the SO neurons. Notably, $\mathrm{PVH}$ and SO OT neurons have different regional input preferences (Fig. 2B). For example, thalamic regions mainly provide inputs to the PVH neurons (Fig. 2A, blue arrowhead), while the striatum (STR) areas (e.g., nucleus of accumbens) provide more input to SO neurons (Fig. 2A, yellow arrowhead). Moreover, the ventral anterior-lateral complex of the thalamus, piriform cortex, and anterior amygdalar area only provide inputs to the $\mathrm{SO}$ neurons. Both the PVH and SO received inputs from the midbrain reticular nucleus (MRN) and the periaqueductal gray (PAG) in the midbrain (MB). The motor-related superior colliculus provides inputs only to the PVH neurons, while the substantia nigra reticular part provides input only to the SO neurons. Notably, PVH OT neurons are strongly connected with the thalamic brain area, which communicates with the cortex and is a key structure for the rapid integration of new learning, working memory, and adaptive decision-making (25), while the SO receives more input from the striatum-like amygdala, which is centered on emotion-based cognitive function (26). This input pattern is overall in agreement with prior reports that utilize traditional retrograde tracers or viral tools $(10,30)$. The anterior two-thirds of the $\mathrm{PVH}$ is known to contain the majority of magnocellular neuroendocrine neurons, while the posterior one-third of the PVH contains the descending preautonomic neurons (8). To investigate potentially segregating input patterns into different PVH subregions, we performed a series of injections with a small volume $(150 \mathrm{~nL})$ of the rabies virus into the anterior-posterior PVH area. However, we did not observe a topographically distinct input pattern into different PVH subregions (Fig. S3). Collectively, we conclude that OT neurons in the PVH received stronger and broader inputs than the SO with distinctive regional input preferences (Fig. 2C). 
bioRxiv preprint doi: https://doi.org/10.1101/2020.10.01.320978; this version posted October 1,2020. The copyright holder for this preprint (which was not certified by peer review) is the author/funder, who has granted bioRxiv a license to display the preprint in perpetuity. It is made available under aCC-BY-NC-ND 4.0 International license.
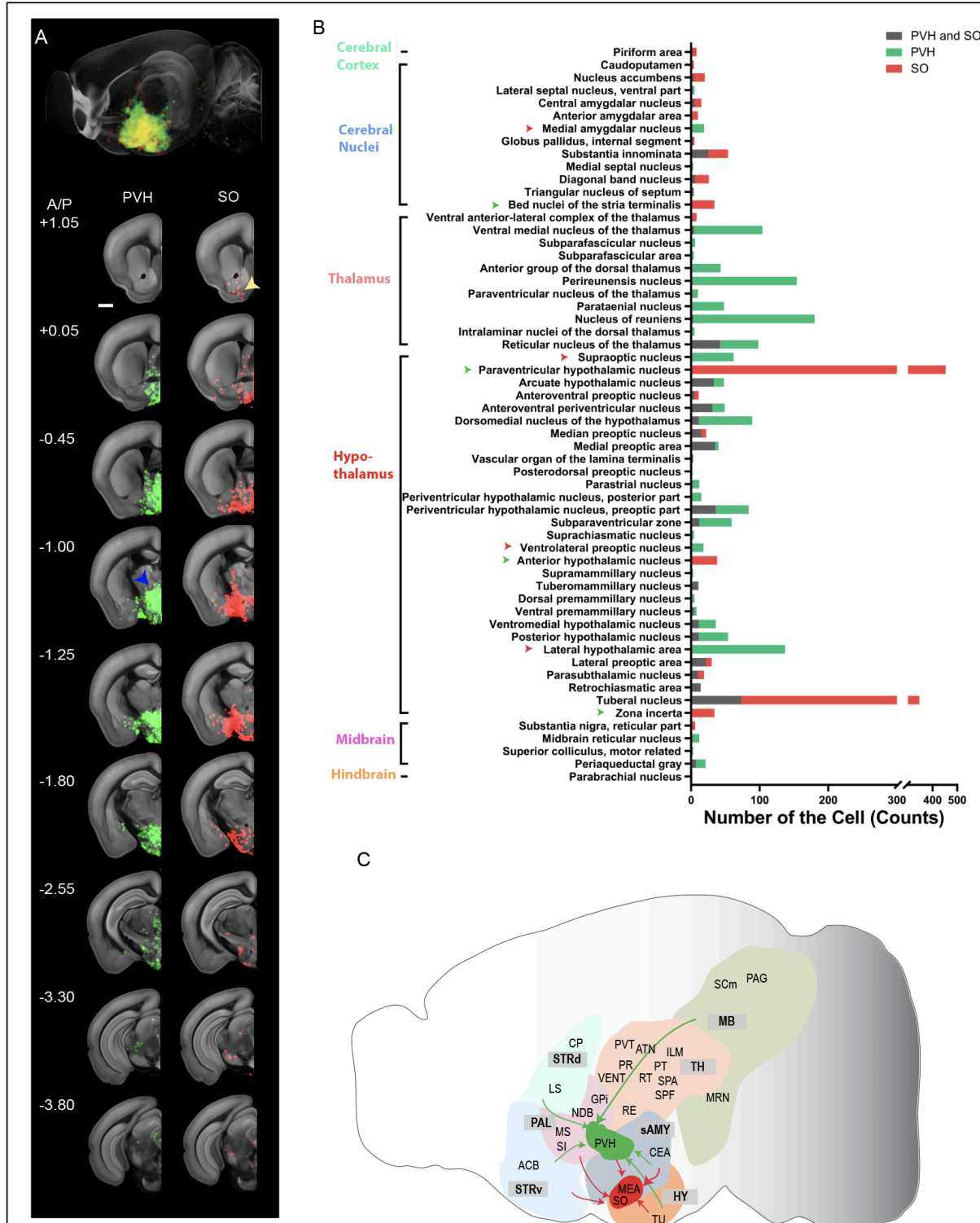

C

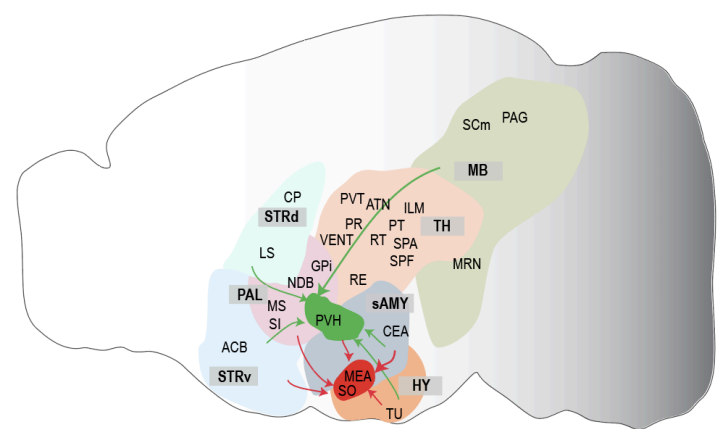

Figure 2. Monosynaptic input map of oxytocin neurons in the PVH and the SO.

(A) Inputs into the PVH (green) and SO (red) OT neurons in representative coronal planes from the Allen $\mathrm{CCF}$. The signals were overlaid on the reference brain. See also Movie S2. Blue and yellow arrowheads indicate unique inputs from the thalamus and the ventral striatum into the PVH and the SO, respectively. Scale bar $=1 \mathrm{~mm}$. (B) Number of cells across anatomical regions providing synaptic inputs into OT neurons in the PVH (green bar) and SO (red bar). The input regions from both PVH and SO were represented as gray. Neighboring brain regions to the PVH and SO with potential signal contamination are not included in the graph, indicated with arrowhead. For example, red arrowhead in the medial amygdala means that input data from the SO was excluded. (C) Schematic input map in the mouse brain. Anatomical brain regions were segmented with different color code and arrows represent input brain regions (green: PVH and red: SO neurons). The full name of abbreviations can be found in Table S2. 
OT neurons can secrete OT through axonal and dendritic processes in coordinated or independent compartment-specific manner $(33,34)$. To visualize the entire central projection patterns of OT neurons, we injected Cre-dependent adeno associated virus 2 (AAV2-CAG-FlexEGFP) in multiple subregions of the PVH and the SO of Ot-Cre knock-in mice. Long-range projection signals from multiple injections were registered onto a reference brain and merged to represent efferent output from each anatomical area (Fig 3A, Movie S3). First, we observed that OT neurons in the PVH project to the HY, the TH, the STR, and the pallidum (PAL), as well as the posterior region including the $\mathrm{MB}$ and the hindbrain (HB) (Fig 3A-B). In contrast, the SO OT neurons project to very limited areas (e.g., pons) in the brain (Fig 3A-B). Quantitatively, OT neurons in the $\mathrm{PVH}$ project to over 99 brain regions including sparse projection to forebrain areas $(\mathrm{N}=3$ males and 3 females, Fig 3B, Table S3). OT neurons in the SO project mainly to hypothalamic regions and sparsely to posterior brain regions including the $\mathrm{PB}$ and the superior olivary complex (SOC) (Fig. 3A-B and Table S3). To examine potential projection topology within the PVH, we targeted PVH subregions by injecting a small volume $(40 \sim 150 \mathrm{~nL})$ of the $\mathrm{AAV}$ virus injection along different $\mathrm{A} / \mathrm{P}$ locations. We did not observe a strong regional projectome difference within the PVH (Fig. S4).

\section{Comparison of input-output connection of oxytocin neurons}

Next, we systematically compared brain regions connected with OT neurons in the PVH and the SO either unidirectionally or bidirectionally (Fig. 3D-E). In the PVH, selected hypothalamic areas (e.g. the TU, the lateral hypothalamic area, and the arcuate hypothalamic nucleus) and the MB (e.g. the PAG) show reciprocal input-output connection. Moreover, the thalamic area (e.g. the nucleus of reuniens, the parataenial nucleus, the ventral medial nucleus of the thalamus, and the reticular nucleus of the thalamus) has strong input to the PVH OT neurons while the MRN shows a stronger output pattern (Fig. 3D). In the SO, OT neurons have dominant input from the $\mathrm{PVH}$ and reciprocal connection with the TU (Fig. 3E). To further understand the input-output relationship quantitatively, we calculated relative input-output strength within the brain regions bidirectionally connected with OT neurons and plotted a log scale of relative input-output ratio (Fig. 3F-G). For example, log-ratio ' 0 ' means that the relative strength of input and the output to that specific region is similar. In PVH OT neurons, many thalamic and hypothalamic regions show input preference and cerebral nuclei have balanced input-output log-ratios while MB regions have output preference (Fig. 3F). In contrast, the SO OT neurons show stronger input preference largely due to their limited projection areas (Fig. 3G). These input-output patterns indicate that after integrating cognitive and physiological information from relatively closer regions (e.g., the $\mathrm{TH}$, the $\mathrm{HY}$ ), $\mathrm{PVH}$ OT neurons broadly project to other brain regions from the forebrain to the brain stem to exert fast and site-specific neuronal modulation (Fig. 3F). In contrast, OT neurons in the SO mainly receive emotion-based cognitive information from cerebral nuclei (e.g., the PAL) and social-sexual information from hypothalamic area while mainly providing limited output to the $\mathrm{HY}$ and posterior pituitary to regulate metabolism and autonomic body functions (Fig. 3G). 


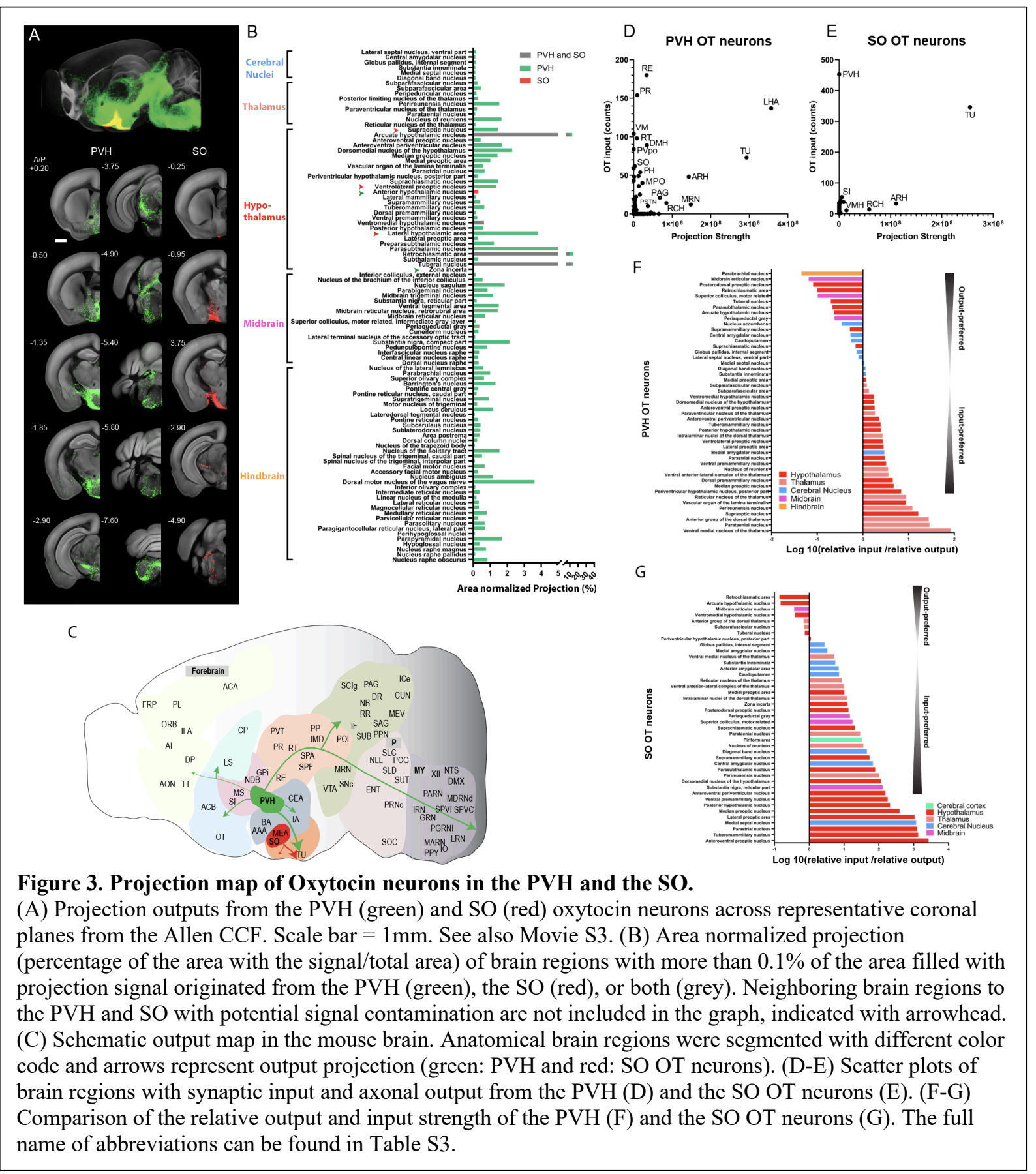

\section{Comparison of oxytocin output and oxytocin receptor expression}

The actions of OT are transduced by a single subtype of the OTR, a seven-transmembrane protein (1). Previous studies suggested a mismatch between presynaptic OT projection and postsynaptic OTR expression $(3,34)$, yet quantitative brain-wide comparison has not been conducted. Previously, we validated that OTR-Venus mice represent the endogenous OTR expression faithfully (11). We imaged a cohort of adult OTR-Venus mice using serial twophoton tomography and mapped OTR expression in the whole adult brain $(n=14$, green in Fig. 
212 4A). Then, we compared the combined projection pattern of $\mathrm{Ot}$-Cre mice in the PVH and the SO 213 (Fig. 3A-C) and OTR-Venus $(+)$ cell density across the entire brain via image registration onto 214 the reference brain (Fig. 4, Table S4, Movie S4). Overall, the OTR (pseudo colored as green) 215 showed high expression in the cortical area with minimal OT projection, while many midbrain 216 and hindbrain regions (e.g., nucleus of the lateral lemniscus) have strong OT projection (pseudo 217 colored as red) with little OTR expression (Fig 4A-C; Movie S4). Correlation analysis between 218 OT and OTR expression across the whole brain did not result in any significant correlation (Fig 219 4D). Nevertheless, a few brain areas contained both OT projection and OTR expression 220 including the lateral septal nucleus, the central amygdalar nucleus (CEA), the paraventricular 221 nucleus of the thalamus (PVT), the PAG, the SOC, and the facial motor nucleus (Fig. 4B).

222 Moreover, a significant OT-OTR correlation was observed within the thalamic and medullary 223 areas. (Figs. 4D-4F). Thus, our results highlight quantitative and spatial discrepancies between 224 OT and OTR in the mouse brain, suggesting largely indirect OT transmission in the brain. 


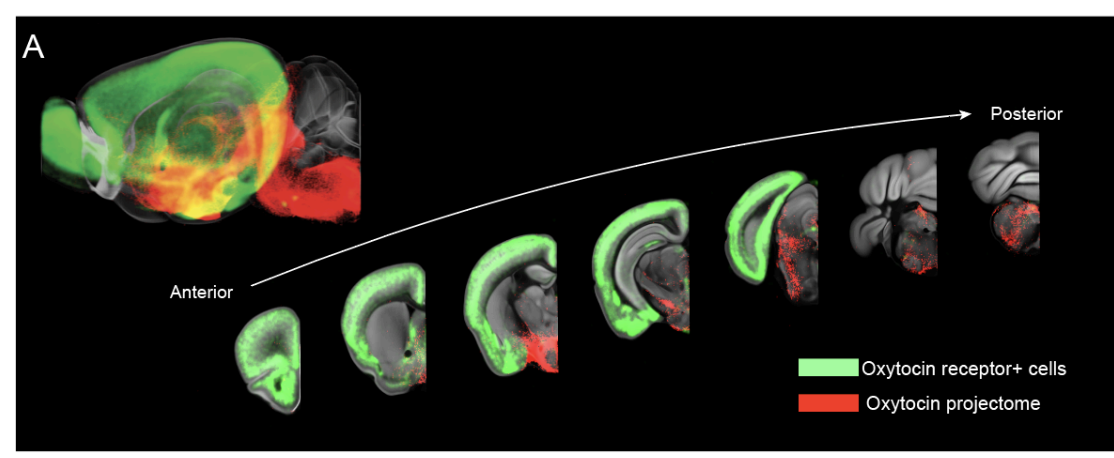

B

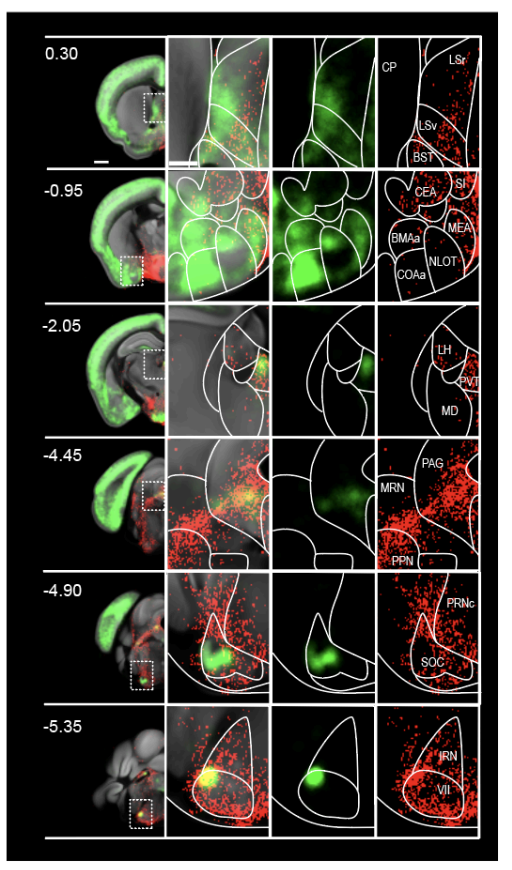

C

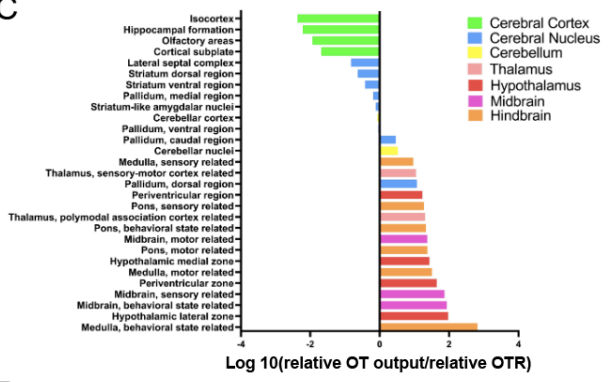

$\mathrm{D}$

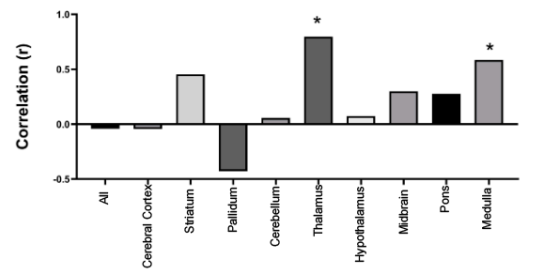

$E$

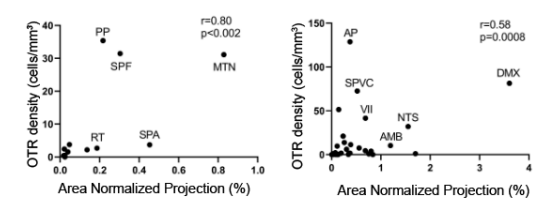

G

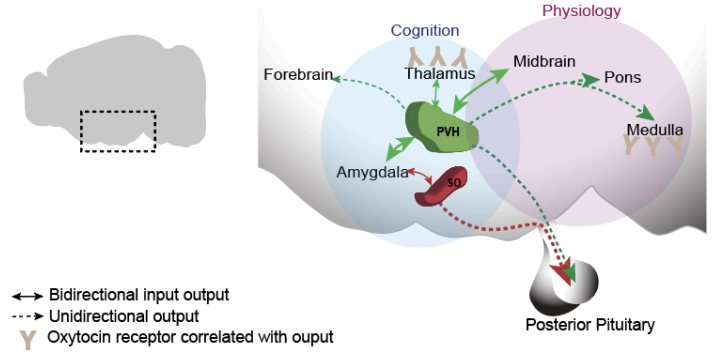

Figure 4. Comparison between the oxytocin output and oxytocin receptor expression.

(A) Composite images of representative OT neuronal projection outputs (red: combined from both the PVH and the SO) and OTR expression (green) across the mouse brain. See also Movie S4. (B) Representative images to show the co-expression of oxytocinergic fibers and oxytocin receptors. White lines represent anatomical borders from the Allen CCF. Scale bars in the column $1=1 \mathrm{~mm}$ and the column 2-4=500 $\mu \mathrm{m}$. (C) Quantitative comparison of relative OT projection pattern and OTR expression. Note that cerebral cortex has very small OT/OTR ratio while the hindbrain and the midbrain shows higher ratio. (D) Correlation between OT projection and oxytocin receptor density (Spearman nonparametric correlation, *: $\mathrm{p}<0.05$ ). Note the significant correlation in the thalamus and the medulla. (E-F) Correlation of brain regions with axonal projection and OTR expression in the thalamus (E) and the medulla (F). (G) Schematic summary of synaptic input and axonal output connectivity of OT neurons and correlated OTR in the mouse brain (green: PVH and red: SO OT neurons). All abbreviations for brain regions can be found in Table S4. 


\section{Discussion}

228 The wiring diagram of the brain is a structural foundation to decipher neural circuits underlying brain function. Here, we present a comprehensive anatomical and connectivity map of the hypothalamic OT neurons and their relationship with postsynaptic OTR expression in the whole mouse brain. We find that OT neurons broadly project to brain regions from the forebrain to the brain stem, while the majority of inputs to OT neurons comes from the thalamus and the hypothalamus. Moreover, we confirm the quantitative mismatch between OT projection and

234 OTR distribution in the brain.

235 OT neurons are mostly located in hypothalamic nuclei with a complex 3D shape (8). To examine OT expression intuitively and quantitatively, we devised a 2D flatmap for OT containing hypothalamic regions from an Allen CCF based reference brain while incorporating anatomical labels from the Allen Institute and Franklin-Paxinos $(23,27)$. This approach allows for the interpretation of OT anatomical location from two independently created and commonly used atlases (28) and provides an alternative coordinate system to understand anatomical connectivity. Our transgenic labeling approach using OT:Ai14 mice revealed that OT neurons in PVH largely maintain their expression throughout adulthood whereas other brain regions (e.g., SO, MEA) showed significant down-regulation of OT expression. Considering the significant role OT signaling plays in shaping the brain development including neural connectivity (35), this transient OT expression in non-PVH areas may provide extra OT signaling to promote early brain development.

Our OT input-output wiring diagram provides brain-wide insights about connectivity-based brain function, which can be divided into two groups (Fig. 4G). The first group contains the brain regions with unidirectional OT output. This pattern is mainly observed in the midbrain, pons, and medulla which are known to be associated with physiological function including body metabolism, sleep, and motor sensory control. For example, the nucleus of the solitary tract (NTS) in the medulla is a part of the neural circuit for food intake and energy expenditure (36). OT neurons in the PVH directly communicate with the NTS to modulate visceral afferent transmission (37). The second group contains brain regions with reciprocal input-output connections with OT neurons including the hypothalamus, thalamus, and striatum that regulate cognitive brain functions such as sexual behavior, memory, attention, fear, reward, and pain. For instance, reciprocally connected regions in the hypothalamic area are highly related to the parental and sexual behaviors such as the MPO (38). Although OT neurons can be further divided into magno- and parvo-cellular neurons, a recent study in rats showed that both cell types in the PVH receive overall similar inputs despite small differences (10). Moreover, we did not observe topographically distinct subregions within the PVH based on input-output maps. This is consistent with a previous retrograde mapping study from the spinal cord, showing that spinal cord projecting OT neurons are distributed across the entire A/P axis (8).

264 Our data also provides a brain-wide perspective on the spatial relationships between OT central 265 projections and oxytocin receptor expression (Fig. 4G). Although the spatial discrepancy of OTOTR was noted before, recent studies showed that most OTR expressing areas contain at least sparse OT projection $(12,18)$. Nonetheless, our analysis showed that there is overall no significant quantitative correlation between OT and OTR across entire brain regions. For example, the cerebral cortex area contains abundant OTR with little to no OT axons. However, OT can still mediate sensory stimuli in the cortex to modify mouse behavior (39). Previous 
271 studies suggest that OTR neurons in the isocortex may receive OT signals indirectly from

272 ventricular pathways via cerebral spinal fluid with delayed and long-lasting effects $(40,41)$. In

273 addition, OT axonal ends in the CEA exert a direct effect on fear suppression with delayed

274 electrophysiological responses ( $\sim$ seconds) in the CEA, suggesting that OT is being released in a

275 non-synaptic way $(18,42)$. In contrast, we also found few areas with high levels of both OTR

276 and OT projection such as the PVT which is involved in maternal behavior (43) and the

277 ventrolateral periaqueductal gray that is implicated in for social defeat stress (44). OT signaling

278 in these areas is likely to occur as the spatially and temporally precise synaptic transmission.

279 Another noteworthy OT-OTR discrepancy is brain regions with abundant OT projection without 280 OTR expression such as sensory related hindbrain areas. Although OTR is a main OT receptor,

281 OT can bind to another receptor to exert its effect. For example, OT can elicit TRPV1 activity in 282 the spinal cord to modulate nociception (45). Non-canonical pathways like this are an under

283 explored mechanism of OT modulation in the central nervous system that requires further study.

284 In summary, our study provides an anatomical foundation to understand diverse functions based 285 on OT neurons in the brain. We deposit all high-resolution imaging data in publicly accessible

286 databases and our website to facilitate data mining. We envision that this OT wiring diagram

287 with quantitative expression data will guide future studies to understand circuit-based

288 mechanisms of OT function and its changes in various brain disorders such as autism. 


\section{Material and Method}

Animals

All animal care and experimental procedures are approved by the Penn State University Institutional Animal Care Use Committee (IACUC). Ot-Cre mice (24) were originally produced in the Gloria B. Choi lab at the Massachusetts Institute of Technology and imported to the Penn State University (Kim Lab). To generate OT:Ai14 mice, Ot-Cre mice were crossed with Ai14 mice, expressing tdTomato following Cre-mediated recombination (Jax: 007914, C57B1/6 J background). Mice received food and water ad libitum and were housed under constant temperature and light conditions ( $12 \mathrm{hrs} \mathrm{light} \mathrm{and} 12 \mathrm{hrs} \mathrm{dark} \mathrm{cycle).}$

\section{$\underline{\text { Stereotaxic surgery and virus injections }}$}

$\mathrm{Ot}$-Cre mice (8-11 weeks old, males and females) were anesthetized with isoflurane (controlled with Somnosuite, Kent Scientific) and mounted on a stereotaxic instrument (Angle Two, Leica) with a heating pad placed underneath. All injections were performed with pulled micropipettes (VWR, Cat\# 53432-706). Through the small opening of the micropipette, virus was delivered at a rate of $75-100 \mathrm{~nL}$ per minute. The speed and volume of injection were monitored along with the calibration marks on the micropipette $(1 \mathrm{~mm}=100 \mathrm{~nL})$. To target the $\mathrm{PVH}$, coordinates are anteroposterior (AP) from the Bregma: $-0.58 \mathrm{~mm}$; mediolateral (ML): $0.27 \mathrm{~mm}$; dorsoventral (DV): $-4.75 \mathrm{~mm}$. Anterior PVH and posterior PVH injection coordinates are $-0.35 \mathrm{~mm}$ (AP), 0.3 $\mathrm{mm}$ (ML), and $-4.5 \mathrm{~mm}$ (DV) and $-0.94 \mathrm{~mm}$ (AP), $0.39 \mathrm{~mm}$ (ML), and $-4.55 \mathrm{~mm}$ (DV), respectively. Coordinates for the SO are $-0.66 \mathrm{~mm}$ (AP), $1.3 \mathrm{~mm}$ (ML), and $-5.8 \mathrm{~mm}(\mathrm{DV})$. For anterograde tracing, 50-500 $\mathrm{nL}$ of AAV2-CAG-Flex-EGFP virus (titer $3.7 \times 10^{12} \mathrm{vg} / \mathrm{ml}$, purchased from UNC vector core) was injected into the $\mathrm{PVH}$ ( $500 \mathrm{~nL}$ for maximum coverage, $50-150 \mathrm{~nL}$ for PVH subregion) and $150 \mathrm{~nL}$ of the virus was injected into the SO. Mice were euthanized three weeks later with Ketamine $(100 \mathrm{mg} / \mathrm{kg})$ and Xylazine $(10 \mathrm{mg} / \mathrm{kg})$ mixture. For monosynaptic retrograde labeling, 50-500 nL of rAAV1-svnp-DIO-STPEPB (titer $3.9 \times 10^{12}$ $\mathrm{cg} / \mathrm{ml}$, purchased from UNC vector core, a gift from Ian Wickersham (46), Addgene plasmid \# 52473 ; http://n2t.net/addgene:52473 ; RRID:Addgene_52473) was injected into the PVH, followed by the same quantity of EnvA G-deleted Rabies-mcherry virus (titer: $8.12 \times 10^{8}$ transduction unit (TU) /ml, purchased from the Salk Institute Viral Vector Core, a gift from Edward Callaway (47), Addgene plasmid \# 32636 ; http://n2t.net/addgene:32636 ; RRID:Addgene_32636) three weeks later into the same location. Mice were euthanized 7-8 days later with Ketamine $(100 \mathrm{mg} / \mathrm{kg})$ and Xylazine $(10 \mathrm{mg} / \mathrm{kg})$ mixture.

\section{$\underline{\text { STPT imaging and related data analysis }}$}

Transgenic or virus injected mice were transcardially perfused with $4 \%$ paraformaldehyde (PFA) in $0.1 \mathrm{M}$ phosphate buffer ( $\mathrm{PB}, \mathrm{pH} 7.4)$ after $0.9 \%$ saline. Brains were dissected out and postfixed in $4 \%$ PFA overnight at $4^{\circ} \mathrm{C}$. Fixed brains were stored in $0.05 \mathrm{M}$ phosphate buffer at $4^{\circ} \mathrm{C}$ until imaged. To image the entire brain, a serial two-photon tomography (TissueCyte 1000; Tissuevision) was used as previously described $(25,48)$. Briefly, the brain was embedded in $4 \%$ oxidized agarose and cross-linked with $0.2 \%$ sodium borohydride solution. The brain was imaged as $12 \times 16 \times 280$ tiles with $1 \times 1 \mu \mathrm{m}^{2} x, y$ pixel resolution in every $50 \mu \mathrm{m} z$-section. We used $910 \mathrm{~nm}$ wavelength for two-photon excitation to excite both green (e.g., eGFP) and red 
signals (e.g., tdTomato). Signals were separated with $560 \mathrm{~nm}$ dichroic mirror and two band path filters (607/70-25 for red and 520/35- 25 for green). Imaging tiles in each channel were stitched with custom-built software (25).

337 For quantitative projection data analysis, we used our previously published pipeline (49). Briefly, both signal and background channels were z-normalized. Then, the background channel images were subtracted from the signal channel images to increase signal-to-noise ratio. Then, projection signals were converted to a binary map by applying an optimized threshold (8x standard deviation) to detect signals while minimizing noise from background autofluorescence. Then, binarized signals in each pixel were counted in $20 \times 20(x, y)$ pixel unit (voxel) and the value was assigned the corresponding voxel across the brain, which is defined as "projection strength". Thus, range of the projection strength in a given voxel is between 0 and 400. Projection strength of each area is calculated by summing up all projection strength within an anatomically defined area. Autofluorescence of brains was used to register each brain to the Allen CCF using Elastix (50), then, the projection signals were transformed to the reference brain. "Area normalized projection" represents normalized occupancy of projection signals in the ROI by dividing the projection strength with a total number of voxels in each ROI. For example, if total voxel count for one ROI was 20,000 and our projection strength showed 2,000 in the ROI, it will be $(2,000 / 20,000) * 100=10 \%$. For cell counting analysis, we used a machine-learning algorithm to detect fluorescently labeled cells (25). The cell density in $2 \mathrm{D}$ (counts $/ \mathrm{mm}^{2}$ ) was calculated by dividing cell number with ROI area. 2D counting numbers were also converted into the $3 \mathrm{D}$ counting using our previously calculated $3 \mathrm{D}$ conversion factor (1.4 for tdTomato) (25). To measure the volume of anatomical ROI, the reference Allen CCF was reversely registered onto individual brains using the Elastix. "Cell density (counts $/ \mathrm{mm}^{3}$ )" was calculated by dividing detected cell numbers in 3D with the anatomical ROI volume. The cell counting analysis was applied to OT:Ai14 and OTR-Venus cell distribution and inputs to the OT neurons.

To compare relative quantity of OT input-output (Fig. 3F-G), bidirectionally connected areas were first selected. Within the selected areas, relative cell density or output data in each region was calculated by dividing each data by summed density or output data from all chosen areas, respectively. Then, $\log 10$ (relative input/relative output) was used to quantify input or output preference of each area. The same approach was used to calculate relative abundance between 364 OT output and OTR expression in Fig. 4C.

To create a representative connectivity map for each anatomical area, we microinjected OT neurons with a large quantity of virus into subregions within an anatomically defined area. Then, the maximum signal of several (4-6) brains from each ROI registered in the reference was used for final analysis to cover entire target anatomical areas for OT input and output data in Figures 2-4, and Movies S2-S4.

373 To determine the correlation between OT area normalized projection and OTR density, we first tested for the normality of the data using the D'Agostino-Pearson normality test. Based on the normality test result, we performed Spearman nonparametric correlation test. GraphPad Prism 8 was used for all statistical analysis and graphs. 
376 To generate the hypothalamic flatmap, we adapted the previously used method (25) and applied

377 it to the hypothalamic region. First, we created a binary image in the hypothalamic area based on

378 the oxytocin expression. Second, a zero line was placed to generate evenly spaced bins along the

379 dorsal to the ventral direction of the PVH and laterally extended to include TU and MEA at

380 different coronal plains. To capture signals on the flatmap, bins were registered into the reference

381 brain and the cell number in each bin was quantified as described before in the STPT data

382 analyses section. Lastly, the mean number of the OT neurons in 8 OT:Ai14 brains were plotted

383 in each flatmap using a custom-build matlab code. For the PVH flatmap, we followed the same

384

385 procedure to generate a hypothalamic flatmap except for bin generation. Instead of delineating bins in a binary image, we assigned bin numbers in the PVH subregion of Franklin-Paxinos atlas (27) along the dorsal to the ventral direction.

\section{Immunohistochemistry, microscopic image, and cell counting}

For immunohistochemistry, fixed brains were either embedded in 3\% agarose or frozen after sinking in 30\% sucrose in $0.2 \mathrm{M}$ Phosphate buffer. Embedded or frozen brains were then cut on a vibratome (Leica vt1000s) or a microtome (Leica SM2010 R) at $50 \mu \mathrm{m}$ thickness. Sections were stored at $-20^{\circ} \mathrm{C}$ in a cryoprotectant solution (30\% sucrose and $30 \%$ glycerol in $0.1 \mathrm{M} \mathrm{PB}$ ) until immunostaining. For oxytocin staining, sections were washed three times in 1x PBS. After 1 hour incubation in blocking solution (10\% donkey serum and $0.1 \%$ Triton X-100), slices were incubated with oxytocin primary antibody (ImmunoStar Cat\# 20068, RRID:AB_572258, 1:1000) in blocking solution for overnight at $4{ }^{\circ} \mathrm{C}$. Sections were then washed three times with $1 \mathrm{x}$ PBS and further incubated in secondary antibodies (Thermo Fisher Scientific Cat\# A-21206, RRID:AB_2535792, 1:500) for 1 hour at room temperature. After washing three times, slices were mounted onto slides and coverslipped with vectashield mounting media (Vector laboratories, H-1500-10). For microscopic imaging, a BZ-X700 fluorescence microscope (Keyence) and a confocal microscope (Zeiss 510) were used. A low magnification objective lens (4x) was used to image with a large enough view to define brain anterior-posterior location from bregma and higher magnification objective lenses $(10 \mathrm{x} \sim 40 \mathrm{x})$ were used to image sections depending on the cell density. Images were delineated manually based on the Franklin-Paxinos 


\section{Acknowledgments}

409 This publication was made possible by an NIH grant R01MH116176 to Y.K. and an NIH grant

410 R24OD18559 to K.C. Its contents are solely the responsibility of the authors and do not

411 necessarily represent the views of the funding agency. We thank Dr. Gloria Choi for kindly

412 sharing OT-Cre transgenic mice and Rebecca Betty for assistance in editing the manuscript. We

413 acknowledge use of computational resources in the High Performance Computing cluster at the

414 Penn State College of Medicine.

\section{Contributions}

419 Conceptualization, Y.K.; Data Collection and analysis, S.S., ; Data Collection, S.M., K.N., M.C., 420 Computer Coding, Y.W.; Web visualization, D.J.V., K.C.; Manuscript preparation: S.S., Y.K 421 with help from the other authors.

\section{Competing Interests}

425 The authors declare no competing interests.

\section{Data Sharing Plan}

429 High-resolution serial two-photon tomography images can be found at https://kimlab.io/brain430 map/ot wiring/

431 Flatmaps and custom-built codes are available at https://kimlab.io/ and can be used without any 432 restriction. 


\section{References}

436

437 1. B. Jurek, I. D. Neumann, The Oxytocin Receptor: From Intracellular Signaling to

438 Behavior. Physiol. Rev. 98, 1805-1908 (2018).

4392 . V. Grinevich, R. Stoop, Interplay between Oxytocin and Sensory Systems in the

$440 \quad$ Orchestration of Socio-Emotional Behaviors. Neuron 99, 887-904 (2018).

441 3. H.-J. Lee, A. H. Macbeth, J. H. Pagani, W. S. Young, Oxytocin: the great facilitator of

442 life. Prog. Neurobiol. 88, 127-151 (2009).

443 4. D. S. Quintana, A. J. Guastella, An Allostatic Theory of Oxytocin. Trends Cogn. Sci.

444 (2020) https:/doi.org/10.1016/j.tics.2020.03.008.

445 5. S. M. Francis, et al., Oxytocin and vasopressin systems in genetic syndromes and

6. K. T. Rajamani, S. Wagner, V. Grinevich, H. Harony-Nicolas, Oxytocin as a Modulator of neurodevelopmental disorders. Brain Res. 1580, 199-218 (2014). Neurosci. 10 (2018).

7. A. J. Guastella, I. B. Hickie, Oxytocin Treatment, Circuitry and Autism: A Critical Review of the Literature Placing Oxytocin into the Autism Context. Biol. Psychiatry (2015).

8. J. Biag, et al., Cyto- and chemoarchitecture of the hypothalamic paraventricular nucleus in the C57BL/6J male mouse: A study of immunostaining and multiple fluorescent tract tracing. J. Comp. Neurol. 520, 6-33 (2012).

9. V. Grinevich, I. D. Neumann, Brain oxytocin: how puzzle stones from animal studies translate into psychiatry. Mol. Psychiatry, 1-15 (2020).

10. Y. Tang, et al., Social touch promotes interfemale communication via activation of parvocellular oxytocin neurons. Nat. Neurosci. (2020) https:/doi.org/10.1038/s41593-0200674-y.

11. K. T. Newmaster, et al., Quantitative cellular-resolution map of the oxytocin receptor in postnatally developing mouse brains. Nat. Commun. 11, 1885 (2020).

12. V. Grinevich, H. S. Knobloch-Bollmann, M. Eliava, M. Busnelli, B. Chini, Assembling the Puzzle: Pathways of Oxytocin Signaling in the Brain. Biol. Psychiatry 79, 155-164 (2016).

13. G. Gimpl, F. Fahrenholz, The oxytocin receptor system: structure, function, and regulation. Physiol. Rev. 81, 629-683 (2001).

14. K. Li, M. Nakajima, I. Ibañez-Tallon, N. Heintz, A Cortical Circuit for Sexually Dimorphic Oxytocin-Dependent Anxiety Behaviors. Cell 167, 60-72.e11 (2016).

15. S. Sabihi, S. M. Dong, N. E. Durosko, B. Leuner, Oxytocin in the medial prefrontal cortex regulates maternal care, maternal aggression and anxiety during the postpartum period.

473 16. N. N. Tirko, et al., Oxytocin Transforms Firing Mode of CA2 Hippocampal Neurons. 
Neuron 100, 593-608.e3 (2018).

17. T. Raam, K. M. McAvoy, A. Besnard, A. H. Veenema, A. Sahay, Hippocampal oxytocin receptors are necessary for discrimination of social stimuli. Nat. Commun. 8, 2001 (2017).

18. H. S. Knobloch, et al., Evoked axonal oxytocin release in the central amygdala attenuates fear response. Neuron 73, 553-566 (2012).

19. V. Ferretti, et al., Oxytocin Signaling in the Central Amygdala Modulates Emotion Discrimination in Mice. Curr. Biol. 29, 1938--1953.e6 (2019).

20. P. J. Ryan, S. I. Ross, C. A. Campos, V. A. Derkach, R. D. Palmiter, Oxytocin-receptorexpressing neurons in the parabrachial nucleus regulate fluid intake. Nat. Neurosci. 20, 1722-1733 (2017).

21. M. Eliava, et al., A New Population of Parvocellular Oxytocin Neurons Controlling Magnocellular Neuron Activity and Inflammatory Pain Processing. Neuron 89, 12911304 (2016).

22. S. Boll, A. C. Almeida de Minas, A. Raftogianni, S. C. Herpertz, V. Grinevich, Oxytocin and Pain Perception: From Animal Models to Human Research. Neuroscience 387, 149161 (2018).

23. Q. Wang, et al., The Allen Mouse Brain Common Coordinate Framework: A 3D

24. H. K. Choe, et al., Oxytocin Mediates Entrainment of Sensory Stimuli to Social Cues of

27. G. Franklin, Keith and Paxinos, The Mouse Brain in Stereotaxic Coordinates (Academic

28. U. Chon, D. J. Vanselow, K. C. Cheng, Y. Kim, Enhanced and unified anatomical labeling for a common mouse brain atlas. Nat. Commun. 10, 5067 (2019).

29. S. Campeau, S. J. J. Watson, Connections of some auditory-responsive posterior thalamic

25. Y. Kim, et al., Brain-wide Maps Reveal Stereotyped Cell-Type-Based Cortical

26. M. P. Madrigal Verdú, S. Jurado, Specification of oxytocinergic and vasopressinergic nuclei putatively involved in activation of the hypothalamo-pituitary-adrenocortical axis in response to audiogenic stress in rats: an anterograde and retrograde tract tracing study combined with . J. Comp. Neurol. 423, 474-491 (2000).

30. M. Cservenák, et al., A Thalamo-Hypothalamic Pathway That Activates Oxytocin Neurons in Social Contexts in Female Rats. Endocrinology 158, 335-348 (2017).

512 33. M. Ludwig, G. Leng, Dendritic peptide release and peptide-dependent behaviours. Nat. 
Rev. Neurosci. 7, 126-136 (2006).

514

515

516

517

518

519

520

521

522

523

524

525

526

527

528

529

530

531

532

533

534

535

536

537

538

539

540

541

542

543

544

545

546

547

548

549

550

34. B. Jurek, I. D. Neumann, The Oxytocin Receptor: From Intracellular Signaling to Behavior. Physiol. Rev. 98, 1805-1908 (2018).

35. T. V Miller, H. K. Caldwell, Oxytocin during Development: Possible Organizational Effects on Behavior. Front. Endocrinol. (Lausanne). 6, 76 (2015).

36. R. A. Travagli, L. Anselmi, Vagal neurocircuitry and its influence on gastric motility. Nat. Rev. Gastroenterol. Hepatol. 13, 389-401 (2016).

37. J. H. Peters, et al., Oxytocin enhances cranial visceral afferent synaptic transmission to the solitary tract nucleus. J. Neurosci. 28, 11731-11740 (2008).

38. M. Gil, R. Bhatt, K. B. Picotte, E. M. Hull, Oxytocin in the medial preoptic area facilitates male sexual behavior in the rat. Horm. Behav. 59, 435-443 (2011).

39. B. J. Marlin, M. Mitre, J. A. D’amour, M. V Chao, R. C. Froemke, Oxytocin enables maternal behaviour by balancing cortical inhibition. Nature 520, 499-504 (2015).

40. J. J. Zheng, et al., Oxytocin mediates early experience-dependent cross-modal plasticity in the sensory cortices. Nat. Neurosci. 17, 391-399 (2014).

41. W. B. Mens, A. Witter, T. B. van Wimersma Greidanus, Penetration of neurohypophyseal hormones from plasma into cerebrospinal fluid (CSF): half-times of disappearance of these neuropeptides from CSF. Brain Res. 262, 143-149 (1983).

42. H. S. Knobloch, V. Grinevich, Evolution of oxytocin pathways in the brain of vertebrates. Front. Behav. Neurosci. 8, 31 (2014).

43. A. Watarai, et al., The blockade of oxytocin receptors in the paraventricular thalamus reduces maternal crouching behavior over pups in lactating mice. Neurosci. Lett. $\mathbf{7 2 0}$, 134761 (2020).

44. N. Nasanbuyan, et al., Oxytocin-Oxytocin Receptor Systems Facilitate Social Defeat Posture in Male Mice. Endocrinology 159, 763-775 (2018).

45. Y. Nersesyan, et al., Oxytocin Modulates Nociception as an Agonist of Pain-Sensing TRPV1. Cell Rep. 21, 1681-1691 (2017).

46. K. Kohara, et al., Cell type-specific genetic and optogenetic tools reveal hippocampal CA2 circuits. Nat. Neurosci. 17, 269-279 (2014).

47. F. Osakada, et al., New rabies virus variants for monitoring and manipulating activity and gene expression in defined neural circuits. Neuron 71, 617-631 (2011).

48. T. Ragan, et al., Serial two-photon tomography for automated ex vivo mouse brain imaging. Nat. Methods 9, 255-258 (2012).

49. M. Jeong, et al., Comparative three-dimensional connectome map of motor cortical projections in the mouse brain. Sci. Rep. 6, 20072 (2016).

50. S. Klein, M. Staring, K. Murphy, M. A. Viergever, J. P. W. Pluim, elastix: a toolbox for intensity-based medical image registration. IEEE Trans. Med. Imaging 29, 196-205 (2010). 
bioRxiv preprint doi: https://doi.org/10.1101/2020.10.01.320978; this version posted October 1,2020. The copyright holder for this preprint (which was not certified by peer review) is the author/funder, who has granted bioRxiv a license to display the preprint in perpetuity. It is made available under aCC-BY-NC-ND 4.0 International license.

\section{Supplementary Information}

553

554

555

556

557

558

\section{Wiring diagram of the oxytocin system in the mouse brain}

Son et al., 


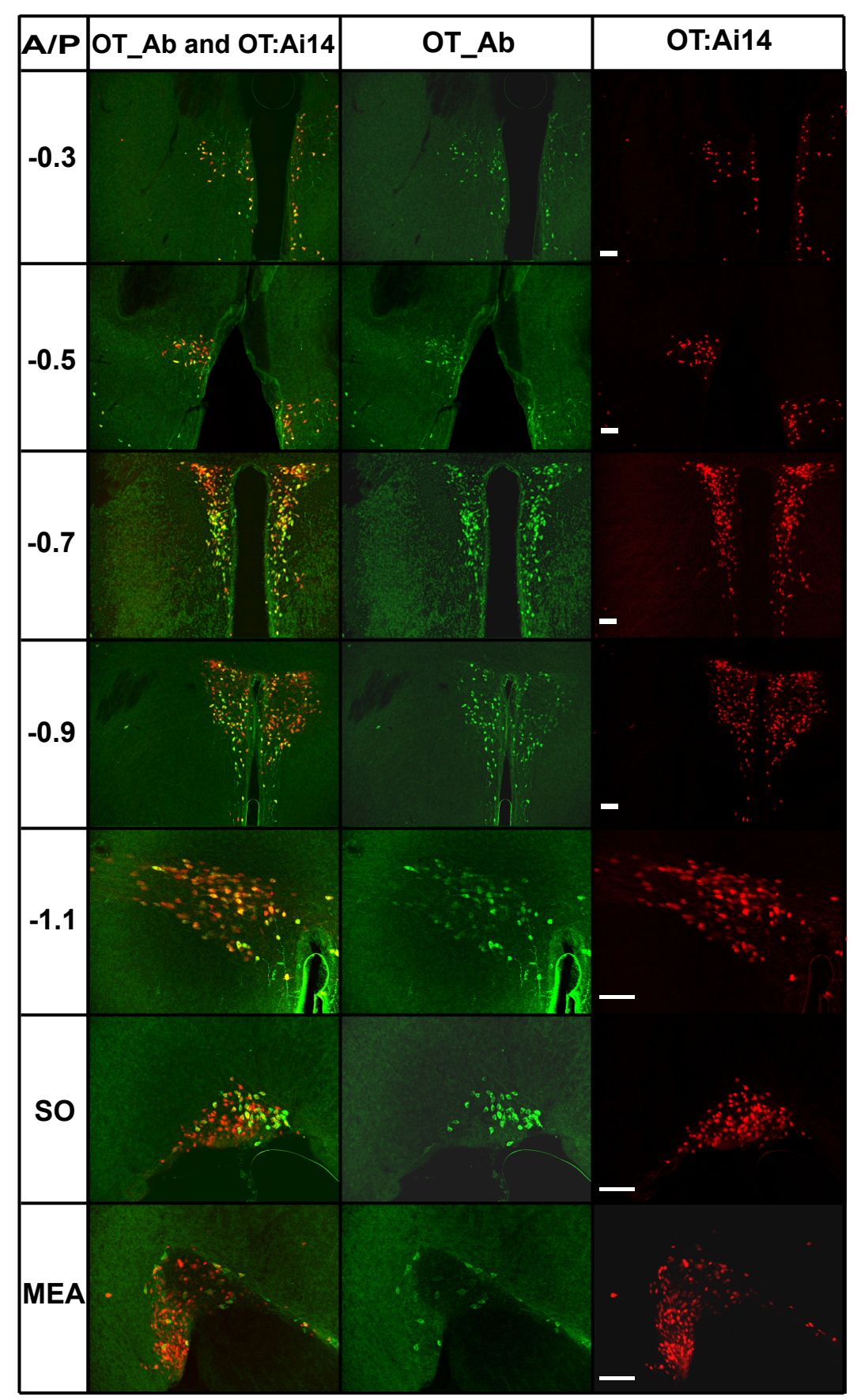

Figure S1. Fluorescent images across 5 levels of the PVH, SO, and MEA. Genetically expressed 563 oxytocin neurons (OT:Ai14) are red and oxytocin immuno staining cellar are labeled with green 564 fluorescent marker are green. Scale bar $=50 \mu \mathrm{m}$. 
A

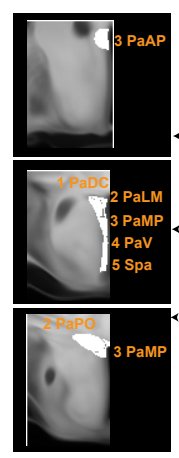

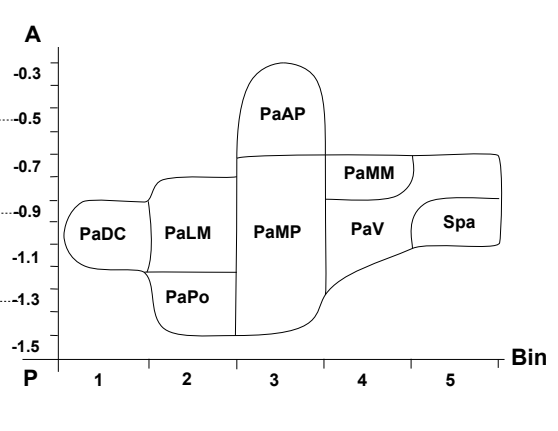

B

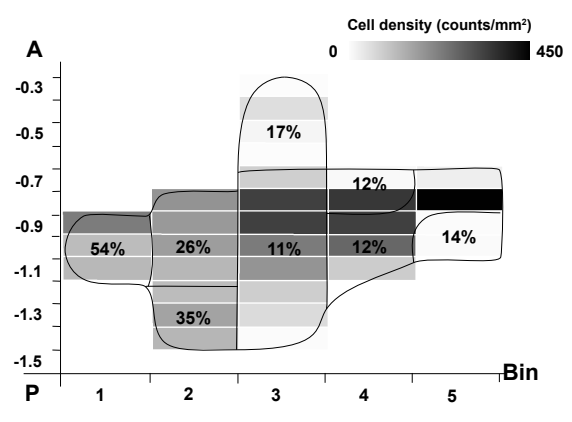

Figure S2. (A) 2D PVH flatmap. Small inserts with coronal sections illustrate the binning system. Bin numbers were assigned on the subregion of $\mathrm{PVH}$ at different coronal planes in number in each brain region represents the percentage of developmentally expressed neurons. The $\mathrm{X}$-axis is for bin numbers and $\mathrm{Y}$-axis is for the bregma $\mathrm{A} / \mathrm{P}$ axis. 

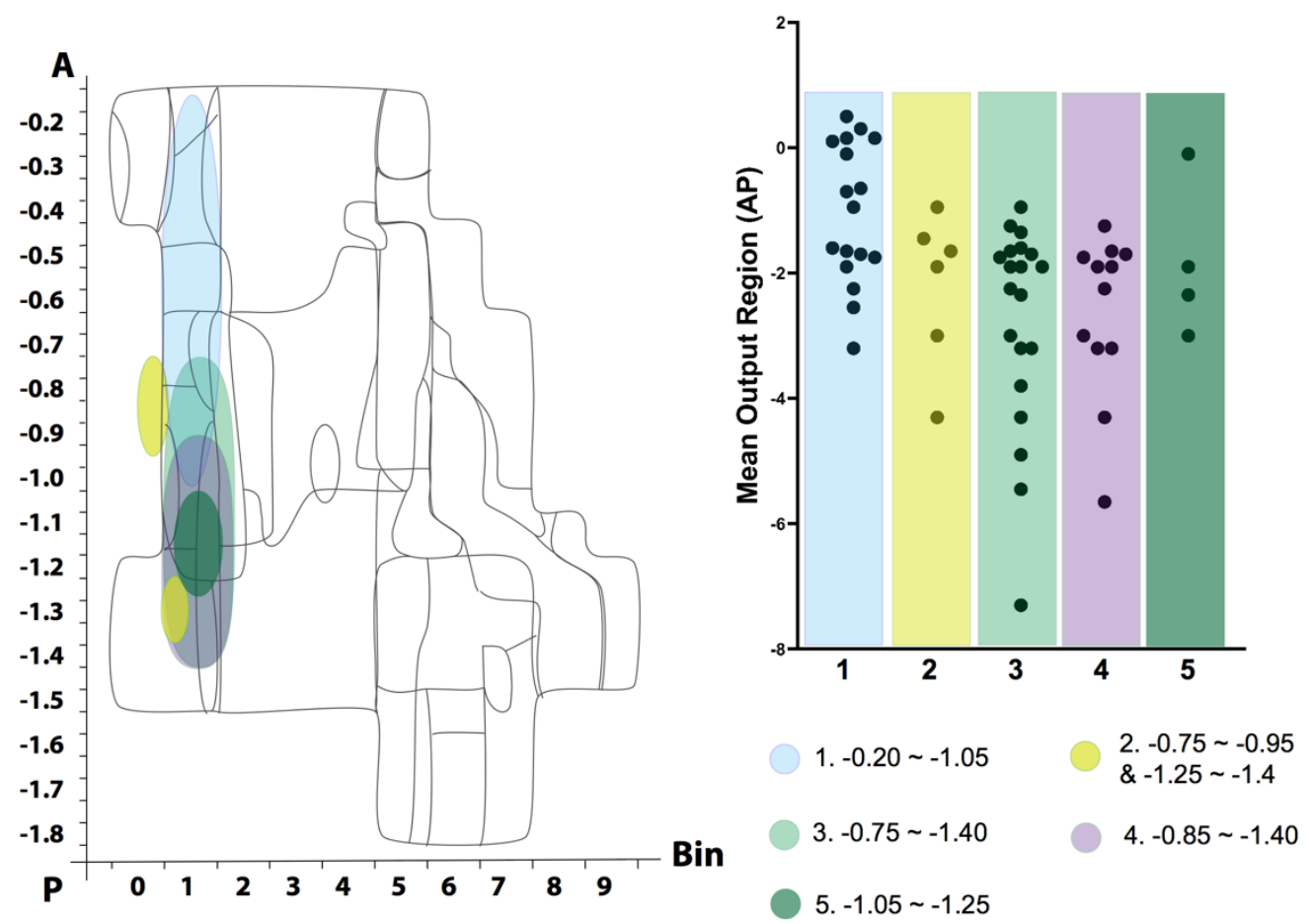

Fig S3. PVH subregion infection. Left: infection coverage on the 2D hypothalamic flatmap. 

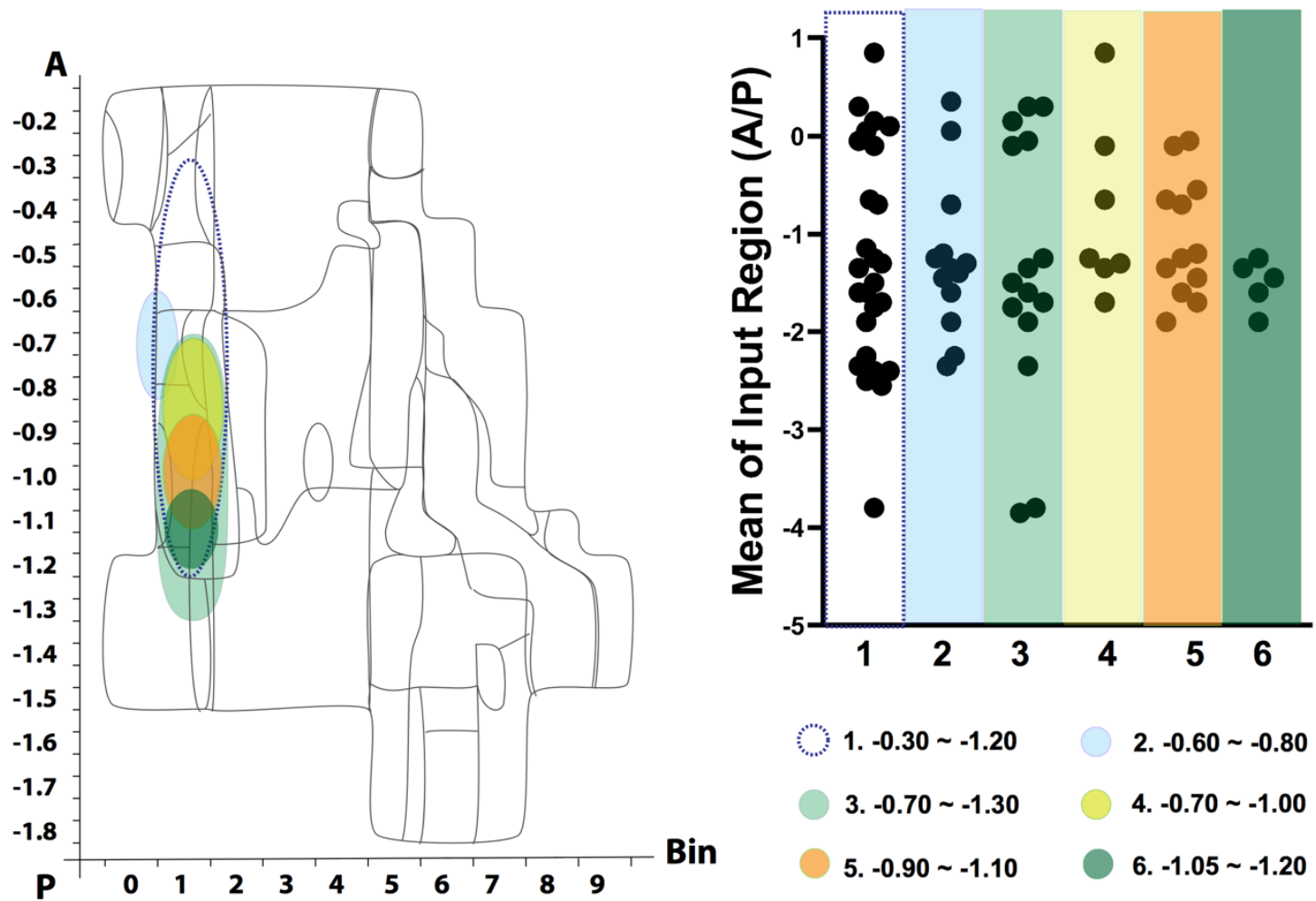

Fig S4. PVH subregional topology. Left: infection area of five different injection on the 2D 


\section{Supplementary Tables}

Table S1. Oxytocin neuronal distribution in the mouse brain

Table S2. Number of cells in brain areas providing monosynaptic input to oxytocin neurons

Table S3. Projection percentage of brain regions with oxytocin neuronal projection

\section{Supplementary Movies}

Movie S1. Oxytocin neuronal expression in the whole brain

Movie S2. Monosynaptic input to oxytocin neurons in the PVH and the SO

Movie S3. Brain-wide projection of oxytocin neurons in the PVH and the SO

Movie S4. Comparison between oxytocin receptor expression and projection of hypothalamic oxytocin neurons. 